\title{
Preference and the Contextual Basis of Ideals in Judgment and Choice
}

\author{
Douglas H. Wedell and Jonathan C. Pettibone \\ University of South Carolina
}

\begin{abstract}
Four experiments assessed preferences for schematic faces. In Experiment 1, eye gap and nose width were manipulated separately, and effects of shifting the range of values were assessed. Descriptive ratings of width showed contrast effects in accordance with A. Parducci's (1995) range-frequency theory. Evaluative ratings of pleasantness showed reversals of preference ordering that were modeled as shifts in ideal points toward the means of the contextual distributions. In Experiments 2 and 3, similar effects of context on preference were demonstrated in a trinary-choice task in which faces varied only in eye gap. In Experiment 4 , eye gap and nose width were manipulated together, resulting in systematic contextual shifts of the ideal face within the 2 -dimensional attribute space. The results demonstrated the pervasive effects of context on the construction of ideals determining preference and underlying attitudes.
\end{abstract}

In many preference domains, more is better. For example, when all other attributes are held constant, increasing monetary gains for an alternative increases preference strength for that alternative. In such domains there is a monotonic relationship between degree of preference and the values on the underlying stimulus dimension. However, a nonmonotonic relationship between preference and value is observed in many other domains, often characterized by a single-peaked preference curve in which the ideal lies at an intermediate value between extremes. For example, with increasing sugar concentration, the pleasantness of the tastes of soft drinks initially increases and then decreases (Moskowitz, Kluter, Westerling, \& Jacobs, 1974), capturing the idea that a drink can be too sweet or not sweet enough. Such single-peaked preference curves are described by Coombs's (1964) ideal-point theory of preference, in which preference is determined by the similarity of the stimulus to an ideal. Monotonic preference curves occur when the ideal is at one extreme or the other, and single-peaked curves occur when the ideal is located at an intermediate value (Coombs, 1964; Coombs \& Avrunin, 1977).

Ideal-point models apply to a wide variety of domains. In addition to the choice domain, perhaps the most notable of these is the attitude domain. Unless the individual holds an ultra-extreme position, the function relating degree of endorsement for an attitudinal position will be single peaked, with the peak representing the ideal position, or the individual's attitude (Eagly \& Chaiken, 1993; Thurstone \& Chave, 1929). Similarly, the structure of categories can sometimes be represented by an ideal-point model, in which the inferred prototype is located at intermediate values of the dimensions

Douglas H. Wedell and Jonathan C. Pettibone, Department of Psychology, University of South Carolina.

This research was supported by National Science Foundation Grant SBR-9319520.

Correspondence concerning this article should be addressed to Douglas H. Wedell, Department of Psychology, University of South Carolina, Columbia, South Carolina 29208. Electronic mail may be sent to wedell@sc.edu. defining the category (Posner \& Keele, 1970; Rosch \& Mervis, 1975). These examples speak to the generality of ideal-point models.

Although single-peaked preferences are well documented, there has been only limited research on how ideals are established. The present research focused on the question of how the recently experienced stimulus context contributes to the determination of ideals, which we believe has important implications for understanding attitude formation and change more generally. Previous judgment research has demonstrated that manipulations of the stimulus distribution can affect ideal-point locations for taste preference (McBride, 1985; Riskey, Parducci, \& Beauchamp, 1979), musical aesthetics (Holbrook \& Anand, 1990), and spatial aesthetics (Baird, Cassidy, \& Kurr, 1978). In these studies, the ideal stimulus value was found to shift toward the mean of the recently experienced set of contextual values. In our investigation we sought to explicate this relationship in several ways. First, we used stimulus materials over which we could exert precise experimental control in order to examine the functional form of this relationship. Second, we examined the generality of context effects on ideals for both judgment and choice tasks and in situations in which the context was manipulated globally by the full set of trials or locally by the current choice set. Finally, we examined how effects found for manipulations of context along a single dimension generalized to contextual manipulations in a multidimensional space. These studies provide insights into the general problem of how our experiences shape our preferences.

\section{Use of Schematic Faces}

We used schematic faces as stimuli because we believed these stimuli represent a reasonable compromise between two sometimes-opposing goals of research: rigor and relevance. The schematic nature of the faces gave us complete control over the facial features so that we could rigorously test precise quantitative theories using psychophysical methods. On the other hand, previous studies of the contextual 
dependence of ideals have used simple sensory dimensions, such as the sweetness of drinks, which may not have much generality to a broader set of more complex social judgments. We felt that evaluations of the pleasantness of these schematic faces could be related to evaluations of the attractiveness of faces more generally. Physical attractiveness has proven to be an important determinant of many social judgments and behaviors (Berscheid \& Walster, 1974), and hence we hoped to broaden the potential implications of our research results. Thus, this set of experiments returns to one of Fechner's original goals, which was the use of the psychophysical method to study precise relationships between aesthetic qualities and physical properties of the stimulus (Boring, 1950). That said, we recognize the limits of generalizing results from judgments of schematic faces to judgments of actual photographs of faces. For example, the extreme features on the schematic faces we used may appear only odd or peculiar, whereas the same distortions of true faces might border on the grotesque or repulsive.

\section{Modeling the Effects of Context}

In the tradition of psychophysics, we modeled the observed responses as a function of the physical attributes of the schematic faces, assuming intervening valuation, integration, judgment, and response processes (Anderson, 1981). We manipulated faces along two dimensions: width of the nose and width of the gap between the eyes. We assumed the psychophysical function relating scale values to physical width was a power function,

$$
S_{\mathrm{i}}=\phi_{\mathrm{i}}^{\mathrm{p}}
$$

where $p$ is the power exponent relating the scale value of stimulus $i\left(\mathrm{~S}_{\mathrm{i}}\right)$ to its physical width $\left(\phi_{\mathrm{i}}\right)$. This function was used in modeling both descriptive and evaluative ratings.

\section{Descriptive Ratings}

Descriptive ratings corresponded to perceptions of the width of the nose or the gap between the eyes. Participants were asked to rate on a 9-point scale how narrow or wide the feature appeared to them. These ratings were modeled by Parducci's $(1965,1995)$ range-frequency theory of judgment, which has provided an excellent description of the effects of contextual manipulations on social judgments, such as judgments of physical attractiveness, equity, happiness, liking, performance, and psychopathology (Mellers, 1983, 1986; Mellers \& Birnbaum, 1983; Smith, Diener, \& Wedell, 1989; Wedell \& Parducci, 1988; Wedell, Parducci, \& Geiselman, 1987; Wedell, Parducci, \& Lane, 1990; Wedell, Parducci, \& Roman, 1989) and psychophysical judgments, such as square size, line length, magnitude of numbers, and numerousity in dot patterns (Birnbaum, 1974; Mellers \& Birnbaum, 1982; Parducci \& Wedell, 1986; Wedell, 1996).

According to the range principle, the rated value of a stimulus is proportional to its location on the subjective scale relative to the minimum and maximum values brought to mind at the time of judgment. If a stimulus fell halfway between the minimum and maximum values, it would be assigned a rating halfway between the minimum and maximum values on the rating scale; that is, a 5 on a 9-point scale. If it fell one quarter of the way up from the lowest end of the range, it would be assigned a value one quarter of the way up from the lowest value on the rating scale; that is, a 3 on a 9-point scale. Thus, the range principle assumes that ratings will be linearly related to the subjective scale values $\left(S_{i}\right.$ 's), with any differences in ratings between context due to shifts in the subjective maximum and minimum values defining the range.

Range-frequency theory asserts that judgments result from a compromise between the use of the range principle and a frequency principle. Differences in the shapes of the distributions of stimuli are modeled by means of the frequency principle rather than the range principle. In the research we present here, the shapes of the frequency distributions of stimuli were not manipulated and so we modeled judgments strictly in terms of the range principle. ${ }^{1}$ Thus, descriptive judgments were fit by the following equation:

$$
\mathrm{C}_{\mathrm{ik}}=a+b\left(\mathrm{~S}_{\mathrm{i}}-\operatorname{Min}_{\mathrm{k}}\right) /\left(\operatorname{Max}_{\mathrm{k}}-\operatorname{Min}_{\mathrm{k}}\right),
$$

where $\mathrm{C}_{\mathrm{ik}}$ is the mean rating of stimulus $i$ in context $k, \mathrm{~S}_{\mathrm{i}}$ is the scale value of the stimulus, $\operatorname{Max}_{\mathrm{k}}$ and $\operatorname{Min}_{\mathrm{k}}$ are scale values defining the subjective range, and $a$ and $b$ are linear constants. Following Parducci (1995), $a$ was assigned the lowest value on the rating scale, and $b$ was equal to the difference between the highest and lowest values on the rating scale.

\section{Pleasantness Ratings}

The other type of rating task in which participants engaged was the rating of how pleasant the facial configurations seemed to them. These ratings reflected their preferences for the different facial configurations or the attractiveness of the faces. We used the following ideal-point model to fit the ratings of pleasantness:

$$
\mathrm{C}_{\mathrm{ik}}=a+b \eta_{\mathrm{i}, \text { ideal }, \mathrm{k}},
$$

where $\mathrm{C}_{\mathrm{ik}}$ is the mean rating of stimulus $i$ in context $k, a$ and $b$ are constants defining a linear response transformation

\footnotetext{
${ }^{1}$ Frequency values are calculated in range-frequency theory using the following equation: $F_{i k}=\left(\operatorname{rank}_{i k}-1\right) /\left(N_{k}-1\right)$, where $F_{i k}$ is the frequency value of stimulus $i$ in context $k$, rank ${ }_{i k}$ is its rank in the stimulus context, and $\mathrm{N}_{k}$ is the total number of stimuli making up the context. Typically, judgments are conceived as a compromise between range and frequency principles, described by a weighted average of range and frequency values as follows: $\mathbf{J}_{i k}=w R_{i k}+(1-w) \mathrm{F}_{i k}$, where $\mathrm{J}_{i k}$ is the internal judgment of stimulus $i$ in context $k$ and $w$ represents the relative weighting of range and frequency values and is often inferred to be close to .5 or an equal weighting of the two principles. In the experiments we report, range and frequency values were highly confounded, so we decided to model the effects of range extension strictly in terms of the range principles as described in Equation 2.
} 
function, and $\eta_{i, \text { ideal, } k}$ describes the similarity of stimulus $i$ to the ideal in context $k$. We modeled single-peaked preference with a gaussian similarity function,

$$
\eta_{\mathrm{i}, \mathrm{idea}, \mathrm{k}}=\exp \left(-\mathrm{d}_{\mathrm{i}, \text { ideal }, \mathrm{k}}^{2}\right) \text {, }
$$

in which similarity between a stimulus and the ideal decreases exponentially as a function of their squared distance within the specified context $\left(\mathrm{d}^{2}\right.$ i,deal,, $\left.\mathrm{k}\right)$. Our general approach followed work by Nosofsky $(1986,1992)$ and defined the squared distance in a euclidean space as follows:

$$
\mathrm{d}_{\mathrm{i}, \mathrm{ideal}, \mathrm{k}}^{2}=c \Sigma w_{\mathrm{m}}\left(\mathrm{S}_{\mathrm{im}}-\mathrm{ideal}_{\mathrm{mk}}\right)^{2},
$$

where $c$ is a discriminability constant, $w_{\mathrm{m}}$ is the weight given to dimension $m, \mathrm{~S}_{\mathrm{m}}$ is the scale value of stimulus $i$ on dimension $m$, and ideal ${ }_{\mathrm{mk}}$ is the value of the ideal point on dimension $m$ in context $k$. When only a single dimension is varied, this equation simplifies to

$$
\mathrm{d}_{\mathrm{i}, \text { deal }, \mathrm{k}}^{2}=c\left(\mathrm{~S}_{\mathrm{i}}-\text { ideal }_{\mathrm{k}}\right)^{2} .
$$

All model fitting was based on variations of Equations 1-6. The quantitative modeling of the data was designed to examine the lawfulness of these relations.

\section{Experiment 1: Manipulating the Range of Attributes in Judgment}

Studies of the nature of physical attractiveness are consistent with a single-peaked preference relationship between facial features and attractiveness. In particular, Langlois and her colleagues (Langlois \& Roggman, 1990; Langlois, Roggman, \& Musselman, 1994) have provided convincing evidence that attractiveness of faces is greatest for faces of average facial configuration. In their experimental paradigm, faces were digitized so that they could be combined by averaging their pixel values into a single composite face. The averaged faces were perceived as more attractive than their constituent faces. This result is consistent with the idea that the prototypical face may be near the average of the population of faces in memory, with attractiveness being highest for faces closest to the prototype or ideal.

The research on averaging of faces has not manipulated context, so it is unclear how the ideal value of a given feature is determined by the recent set of contextual experiences. We examined this question using simplified schematic faces and a design similar to that of Riskey et al. (1979). In Riskey et al.'s study, participants in separate blocks of trials rated the sweetness and pleasantness of a series of soft drinks varying in sucrose concentration. The contextual distribution of sweetness concentrations was either positively or negatively skewed. In Experiment 1 we presented participants with sets of schematic faces and asked them to rate the faces in terms of width of nose, width of eye gap, and pleasantness. Context was varied by shifting the range of nose widths or eye gaps to create low- and high-contextual sets, with seven faces common to the two contexts serving as comparison stimuli.
Participants saw schematic faces like those shown in Figure 1. Each face consisted of the same oval-shaped head, smile, eyes, and eyebrows. Faces differed only in the width of the nose and in the gap separating the eyes. Within a given judgment set, only one of these features was manipulated (i.e., either eye gap or nose width). Stimuli consisted of seven target faces interspersed with seven contextual faces. The low context included faces with a very narrow eye gap or nose width, and the high context included faces with a very wide eye gap or nose width. Participants made descriptive or evaluative ratings of the faces in separate blocks of trials.

Our predictions were straightforward. On the basis of psychophysical research on judgments of magnitude (Parducci \& Wedell, 1986), we predicted contrast effects on the descriptive dimension. The same target face should be rated as having a wider eye gap or a wider nose in the low context than in the high context. Of greater relevance to our focus on ideals, we predicted that pleasantness ratings would follow a crossover interaction corresponding to a shift in the peak of the preference function across contexts. If the ideal value of the attribute corresponds to the attribute level that is judged as moderate or average, then the ideal should shift toward a lower value in the low context and a higher value in the high context. Thus, a face with a moderately narrow nose should be judged more pleasant than a face with a moderately wide nose in the low context, but this relationship should be reversed in the high context. If context effects on descriptive ratings reflect only output tendencies or biases, then we would not expect to find a corresponding shift in ideal points.

\section{Method}

Participants and design. Participants were 102 undergraduates from the University of South Carolina psychology department

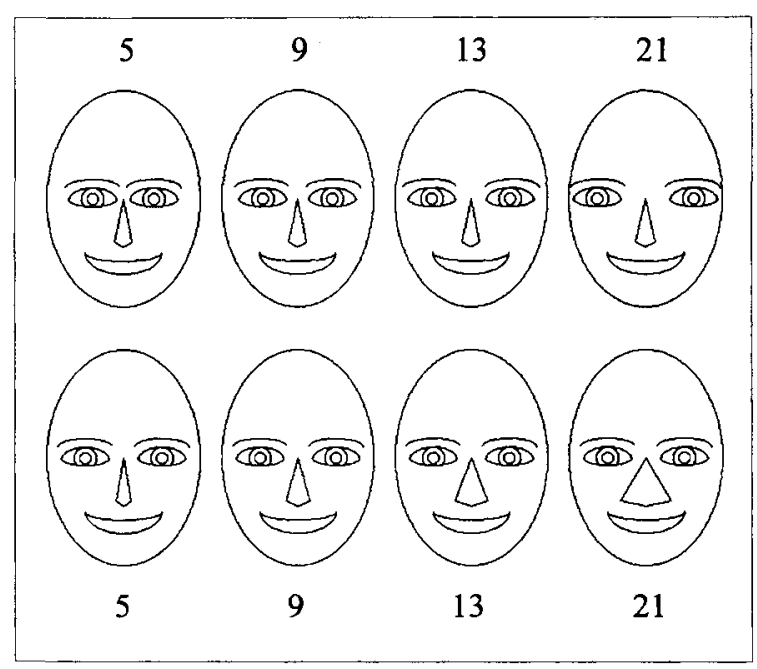

Figure 1. Examples of schematic faces used in Experiment 1. Target Faces 5, 9, 13, and 21 are shown, with eye gap manipulated in the top row and nose width manipulated in the bottom row. 
participant pool who volunteered for this experiment in exchange for course credit. All participants rated two sets of schematic faces, on both descriptive and evaluative rating scales. In one set of schematic faces the width of the gap between the eyes varied, and in the other set the width of the nose varied. Within each set, 7 target faces were judged by all participants. We manipulated context between subjects by including 7 contextual faces of either low or high values in the set for judgment. Other between-subjects variables were the order of presentation of sets (eye gap manipulation or nose width manipulation first) and order of presentation of the judgment tasks within sets (descriptive first or pleasantness first). Participants were randomly assigned to the between-subjects conditions. For each judgment task, the 14 faces constituting a set were each presented in four blocks, with presentation order randomized within blocks. Thus, each face was judged four times on each measure. Dependent variables were the 9-point descriptive ratings of the features and the 9-point evaluative ratings of pleasantness.

Materials and apparatus. All materials and instructions were presented on IBM-compatible computers with 15-in. (38-cm) monitors. Faces were presented in videographics array mode at a resolution of $640 \times 480$ pixels. A norming study $(N=42)$ was used to establish 25 value levels each for the width-of-the-nose domain and the width-of-the-eye-gap domain. Target stimuli consisted of seven faces (value levels of $5,9,11,13,15,17$, and 21). The five moderate target faces were selected to fall close to the predicted ideal point, with the two more extreme faces included to facilitate fitting preference functions to the data. Contextual stimuli consisted of seven faces: value levels of $1,2,3,4,6,7$, and 8 in the low set and 18, 19,20,22, 23, 24, and 25 in the high set.

Examples of four of the seven target faces used in each domain are presented in Figure 1. Each face was 70 pixels wide and 100 pixels long. Nose width was measured in pixels at the widest part of the nose. The narrowest nose was 6 pixels wide, with successive faces increasing in increments of 4 pixels. Eye gap was measured in pixels from the innermost points of the eyes. The smallest eye gap was 4 pixels, with successive faces increasing in increments of 2 pixels. All other features of the faces were held constant across the full set of faces. When eye gap was manipulated, nose width was held constant at 30 pixels (Size 6). When nose width was manipulated, eye gap was held constant at 26 pixels (Size 12).

Procedure. Groups of 1-5 participants were tested at the same time within a laboratory room with computer terminals spaced approximately $1 \mathrm{~m}$ apart. After reading the general instructions for the experiment, participants were presented the instructions for the rating task that they were to perform first. The $\mathbf{1 4}$ different faces for the corresponding task were then previewed, 1 at a time, for $4 \mathrm{~s}$ each. Faces were presented successively in random order and centered on the screen. Following the preview, participants rated the $\mathbf{1 4}$ faces four times each in a block-randomized order, for a total of 56 trials. On each trial, the rating scale appeared at the bottom of the screen, and participants recorded their ratings by entering a corresponding number. After each block of 56 trials, there was a 1 -min break. In the second block of 56 trials, participants rated the same faces, but on a different rating scale. In the last two blocks of 56 trials, participants rated the other set of faces.

Two rating tasks were used. In the descriptive rating task, participants rated the width of the nose or the width of the gap between the eyes of the presented face. Ratings were made on a scale of 1 to 9 , with 1 labeled very narrow and 9 labeled very wide. In the pleasantness rating task, participants were asked to judge how pleasant the nose width or eye gap of the face appeared to them. Ratings were again made on a scale of 1 to 9 , with 1 labeled very unpleasant and 9 labeled very pleasant.

\section{Results}

Using Cronbach's alpha, we used ratings from the four blocks of trials for each rating task to estimate the reliability of the mean ratings. Five participants with reliabilities less than .40 on at least one task were dropped from the analyses reported below.

Descriptive ratings. The top panels of Figure 2 present the mean ratings of size for the target and contextual faces from the two stimulus domains, nose width and eye gap. We conducted repeated-measures analyses of variance (ANOVAs) on the ratings of the seven target faces separately for each domain, with context (low or high), task order (descriptive first or pleasantness first), and set order (eye gap set first or nose width set first) as between-subjects variables. For both sets of stimuli, there was a strong main effect of context, $F(1,89)=122.5, p<.001$, for eye gap stimuli, and $F(1,89)=92.1, p<.001$, for nose size stimuli. As shown in Figure 2, this effect reflects the much higher ratings of the target stimuli in the low-context than in the high-context condition, a difference in mean ratings of 1.2 and 1.3 for eye gap and nose width ratings, respectively. Thus, the descriptive ratings show the usual contrast effect found for ratings of size (Parducci \& Wedell, 1986). The Target $\times$ Context interaction was not significant for the nose size domain but was significant for the eye gap domain, $F(6,534)=3.1, p<$ .05 . This interaction reflects some small differences in the magnitude of contextual effects across targets and is not of particular interest.

Naturally, both sets of stimuli showed a large main effect of target, $F(1,89)=698, p<.001$, for eye gap, and $F(1,89)=972, p<.001$, for nose width, demonstrating that the target faces were highly discernible from each other. A main effect of stimulus presentation order was found for nose width stimuli, $F(1,89)=8.8, p<.01$, reflecting higher overall ratings of nose width when the eye gap stimuli were presented first. The only other interaction involving context was a three-way interaction of context, stimulus presentation order, and the order of the rating tasks for the nose width stimuli, $F(1,89)=7.2, p<.01$. This interaction reflects the fact that context effects were greatest when the nose width set was presented first and descriptive ratings followed evaluative ratings. The three-way interaction represents a shift in magnitude of context effects rather than direction, with significant effects obtained in every cell of the design. Because the interaction was not replicated for eye gap or for evaluative ratings in either domain (see below), it was not considered particularly meaningful. All other effects were nonsignificant.

Evaluative ratings of pleasantness. The bottom panels of Figure 2 present the mean ratings of pleasantness for target and contextual faces from both stimulus domains. The ideal-point structure of the pleasantness data resulted in a correlation matrix of the dependent measure across conditions that was far from homogeneous. There were positive correlations between ratings of similar targets and negative correlations between ratings of dissimilar targets (typical of ideal-point data). Thus we used a repeated-measures multivariate analysis of variance (MANOVA) procedure to ana- 

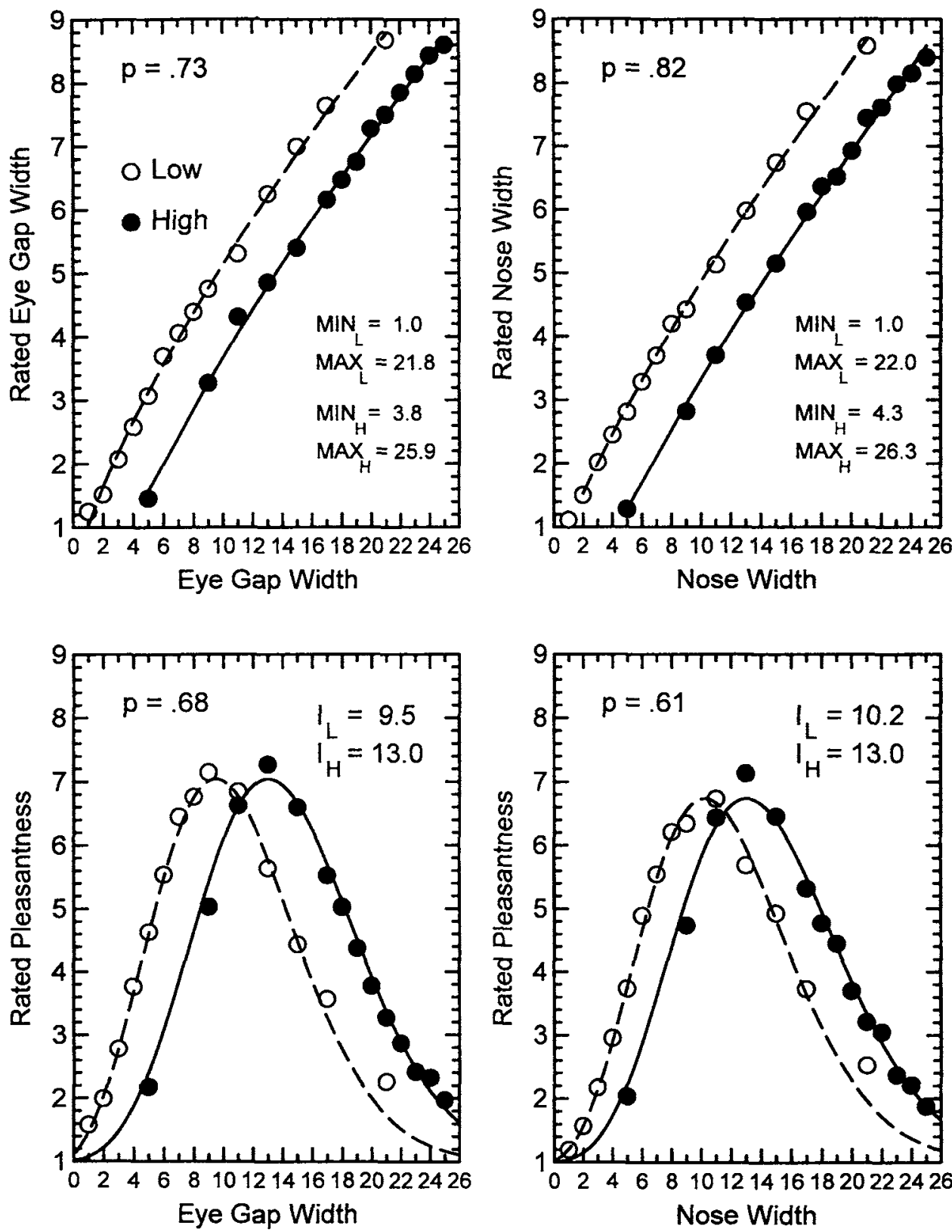

Figure 2. Mean ratings from Experiment 1 segregated by context (low [L] or high [H]), rated dimension (width or pleasantness), and feature manipulation (eye gap or nose width). The data of the top panels are consistent with the subjective range shifting with context. Lines represent the fit of the range-frequency model with fitted parameters for the minimum (MIN) and maximum (MAX) values in low and high contexts. The data of the bottom panels are consistent with preference ideals shifting with context. Lines show the fit of the ideal-point model, with the ideal (I) point shown for low and high contexts. The parameter $p$ corresponds to exponent of the power function relating physical width to subjective width fit to the data in each panel.

lyze the ratings of the target stimuli. We performed separate $2 \times 2 \times 2 \times 7$ repeated-measures MANOVAs on the pleasantness ratings of the target stimuli. The critical test of whether context affects ideal-point location is the Context $X$ Target interaction, which was highly significant for both stimulus domains: $F(6,84)=13.4, p<.001$, for eye gap, and $F(6,84)=6.4, p<.001$, for nose width. In each case, the data were quite orderly. Ratings fell along a singlepeaked function, with ratings rising up to a peak and then falling off again. Ideal points varied systematically with context in both stimulus domains. The ideal face in the high-range context had a wider nose or a wider eye gap than 
the ideal face in the low-range context. This shift in ideal point produced a strong crossover interaction. For example, consider Target Faces 9 and 13, which are pictured in Figure 1. In the low context, Target Face 9 was judged much more favorably on the pleasantness dimension than Target Face 13 for both eye gap ( $M_{9}=7.2$ vs. $\left.M_{13}=5.7\right)$ and nose width $\left(M_{9}=6.4\right.$ vs. $\left.M_{13}=5.8\right)$. In the high context, this relationship was reversed for eye gap $\left(M_{9}=4.8\right.$ vs. $\left.M_{13}=7.2\right)$ and nose width $\left(M_{9}=4.8 \mathrm{vs}\right.$. $\left.M_{13}=7.1\right)$.

No higher order interactions involving the order of stimulus presentation or order of rating task were observed in either domain. The significant main effect of target in both domains simply demonstrated that targets differed in pleasantness: $F(6,84)=29.9, p<.001$, for eye gap, and $F(6,84)=52.9, p<.001$, for nose width. Finally, when the same analyses were run on just the first block of ratings, the same pattern of significance was found, indicating that effects of context were quite clear at the time the faces were first judged.

Analysis of individual ideal points. Another way to examine the data is to estimate ideal points for each individual and analyze how these differ as a function of context. We estimated ideal points using only the seven target faces and the nonlinear procedure described in the Modeling the data section. The pattern of responding of 9 participants in the eye gap domain and 18 participants in the nose size domain did not result in unique solutions, and their data were dropped, leaving 88 participants in the eye gap domain and 79 in the nose width domain. Ideal points were analyzed in $2 \times 2 \times 2$ ANOVAs (with context, stimulus order, and task order as independent variables), and the results were consistent with those reported above. There was a significant main effect of context, $F(1,80)=31.2, p<$ .001 , in the eye gap domain, and $F(1,71)=24.0, p<.001$, in the nose width domain. The means of ideal points from these analyses were close to those based on the fitting of the full set of participants (shown in Figure 2). No other significant effects were found. ${ }^{2}$

Modeling the data. The data of Experiment 1 are very regular, suggesting that they may derive from processes of a highly orderly nature. It is therefore instructive to determine how well existing theoretical models can account for these data. We modeled the descriptive judgments using Equation 2 , with the psychophysical power function of Equation 1 substituted for scale values. Altogether, five parameters were used to fit the data in each domain: Two parameters for each distribution were used to define the range, and a power exponent $(p)$ was used to define the psychophysical function. The fit of the model is shown in the top panels of Figure 2 . As expected, the data are explained by assuming that the minimum and maximum values defining the subjective range shift upward in the high context relative to the low context. The fit of the model was excellent, with the proportion of variance in mean ratings predicted by the model near $1.0\left(R^{2}=.998\right.$ for both eye gap and nose width $)$.

A simple ideal-point model was generated to fit the pleasantness data. The equation that was fit to the data was

$$
\mathrm{C}_{\mathrm{ik}}=1+b\left[\exp \left(-c\left(\phi_{i}^{\mathrm{p}}-\mathrm{ideal}_{k}^{\mathrm{p}}\right)^{2}\right]\right.
$$

The expression within brackets in Equation 7 can vary between 0 and 1 and takes the form of a normal curve. The additive constant was fixed at 1 because this value corresponded to the lowest value on the rating scale. The multiplicative constant, $b$, determines the height of the function when mapping it onto the rating scale. The horizontal location of the function for each context is given by the ideal point for that context $\left(\right.$ ideal $\left._{k}\right)$. Preference falls off as a gaussian function of the distance between the stimulus and the ideal point, with the stimulus's subjective location $\left(\phi_{i}^{p}\right)$ being determined by the psychophysical power function. The $c$ parameter is a discriminability parameter and determines the width or narrowness of the rating function.

The data for each of the two bottom panels of Figure 2 were well described by a five-parameter model in which $p, b$, and $c$ were constrained to be equal across contexts and only ideal point $\left(\right.$ ideal $\left._{k}\right)$ differed with context. Thus, all differences between contexts were captured by variation of a single parameter. The fit of the model was excellent, with the proportion of variance in mean ratings predicted by the model near $1.0\left(R^{2}=.990\right.$ and $R^{2}=.984$ for eye gap and nose width, respectively). This same procedure was used to estimate the ideal points for individual participants, with these ideal-point estimates being used in the analyses described above. ${ }^{3}$

Finally, we can ask about the correspondence between the context effects on the dimensional rating scale and the context effects on the ideal-point locations. To what degree can the ideal-point locations be predicted by assuming the ideal point corresponds to a fixed position on the contextu-

\footnotetext{
2 The failure to fit the model to the data in 27 cases occurred primarily because these were instances in which the function was essentially monotonic within the range of target stimuli. In such cases, the location of the peak of a gaussian function (the ideal point) is arbitrary.

${ }^{3}$ Nosofsky (1992) used both exponential and gaussian similarity functions in fitting identification data, pointing out that gaussian functions tend to be more characteristic of situations in which stimulus discrimination is low. Although the gaussian similarity model clearly fit better than an exponential similarity model at the level of the overall data, it is important to determine if this relationship held for individual data. For example, if several individuals with exponential decay functions are combined together, their combined data may appear to follow a gaussian similarity function. Thus, we fit all participants' target ratings for both eye and nose preference conditions using three-parameter gaussian and exponential decay models, with the fitted parameters being the multiplicative constant (b), the sensitivity parameter (c), and the ideal point. To reduce the number of fitted parameters, the power exponent describing psychophysical function was held constant at $p=1.0$. For each domain, we tallied whether the proportion of variance explained was greater for the exponential or the gaussian model. If one of the two models did not converge, we discounted this comparison. Of the 88 cases in which both models converged for eye gap data, $86 \%$ favored the gaussian model over the exponential model. Of the 79 cases in which both models converged for nose width data, $78 \%$ favored the gaussian model. Thus, the fits to the individual data provided general support for the use of a gaussian similarity function over the use of an exponential decay function.
} 
ally altered dimensional rating scale? For the eye gap domain, the best fit was given by assuming ideals corresponded to a rated value of 4.71 , which yielded ideal-point values of 9.40 and 13.13 for low and high contexts, respectively. For the nose width domain, the best fit was given by assuming ideals corresponded to a rated value of 4.59, which yielded ideal-point values of 9.62 and 13.63 for low and high contexts, respectively. In both cases, the predicted ideal points were close to the ideal points inferred from the pleasantness ratings (see Figure 2). Thus, the data are reasonably well described by a model that places the ideal at a fixed value on the contextually altered subjective scale of dimensional values. The correlation between the mean of the target stimulus ratings on the dimensional scale and the inferred ideal point for each participant was -.58 for eye gap and -.59 for nose width, respectively. These fairly large and significant correlations support a model that assumes contextual shifts in the descriptive judgments lead to corresponding shifts in the locations of ideals determining preference.

\section{Discussion}

The data of Experiment 1 were extremely orderly, as one might expect from judgments based on simple psychophysical materials. The usual contextual contrast effects were obtained on the descriptive dimensions. More important, the perceived pleasantness of these features was well described by a model in which the ideal is contextually determined. Thus, these results replicate and extend work by Riskey et al. (1979) in the taste domain to a domain more relevant to social perception. These results are also consistent with the view that contextual effects on descriptive dimensions can correspond to real shifts in subjective evaluations (Manis, 1967; Wedell, 1995, 1996). One reason psychologists are reluctant to accept such a conclusion is that rating scales are typically assumed to reflect either ordinal or interval measurement of a construct. Thus, an upward shift of values on the rating scale could simply reflect a distortion of the measurement instrument rather than a change in the evaluation of the underlying psychological construct. However, the crossover interactions demonstrated for the preference data are not easily explained away in this manner. If subjective impressions were constant across the two contexts, then participants would be assigning a higher pleasantness rating to a less pleasant face in at least one of the two contexts. This seems implausible, and thus these data strongly support the assertion that the subjective evaluation of a stimulus depends on context.

One conceptualization of these results is that the ideal point is constructed on the basis of one's judgments of the underlying descriptive dimension. As descriptive judgments shift with context, so too will the stimulus defining the ideal point. As a first order of approximation, the ideal corresponded roughly to a value close to 5.0 on the contextdependent descriptive rating scale. Thus, in the low-context condition, Face 9 was rated as more pleasant than Face 13, because the perceived value of its eye gap or nose width was judged closer to the midpoint of the descriptive scale. In the high context, the reverse was true. These effects do not require a large number of exposures, as they were observed within the very first trial block.

The excellent fit of the guassian ideal-point model lends support to the idea that preferences reflect a proximity process in which the current stimulus is compared with an ideal stimulus retrieved from memory (Coombs, 1964). Preference increases as a function of the similarity between the stimulus and the ideal. Although the similarity function is often characterized by an exponential decay function in categorization (Nosofsky, 1992), the preference data of Experiment 1 were better characterized by a gaussian similarity function, at both the group and individual levels. The close fit of the data to a specific functional form suggests that these materials may be particularly useful in future tests of models that predict subtle but precise changes in functional form.

The results of Experiment 1 support the idea that the most attractive facial configurations are those that have features closest to the average of the feature set (Langlois \& Roggman, 1990). In the present framework, this result is interpreted in terms of basic judgmental processes that are sensitive to the contextual distribution. Values near the average of the contextual distribution tend to be judged more moderately and, in accordance with ideal-point theory, moderate values are most preferable (Coombs \& Avrunin, 1977). The contextual dependence of ideals found for the simple schematic faces used in Experiment 1 suggests a mechanism for explaining possible individual and cultural differences in judgments of attractiveness. Presumably, individuals who are exposed to different distributions of faces will establish different ideals. However, the research on cultural differences in perceived attractiveness suggests that there is more agreement than disagreement in judgments of attractiveness across cultures (Bernstein, Lin, \& McClellan, 1982; Cunningham, Roberts, Barbee, Druen, \& Wu, 1995; Thakerar \& Iwawaki, 1979). It may be that effects found for simple schematic faces simply do not generalize to complex, real world faces. Alternatively, the average of facial features across cultures may be rather similar so that similarities in ratings outweigh differences. Yet another possibility is that when features are manipulated in combination, the effects of context are not as clearly determined as when isolated features are manipulated. We tested this position in Experiment 4.

\section{Experiment 2: Context Effects in Choice}

Experiment 1 demonstrated that repeated exposure to a series of contextual faces quickly and systematically altered pleasantness relations among those faces. In Experiment 2, we examined whether these effects generalize to choice. Participants, presented with one of two sets of faces, selected the face they most preferred. Faces varied only in the gap between the eyes. In the low-range set, two target faces ( 9 and 13) were presented along with a narrow-gap face (1). In the high-range set, a wide-gap face (21) was substituted for the narrow-gap face. If contextual effects occur immediately, then the ideal point should differ for the 
two sets, with Face 9 being preferred to Face 13 in the low-range set but the reverse being true in the high-range set.

To get a feel for the procedure used in Experiment 2, cover the rightmost face of the top row of Figure 1 and consider the pleasantness of the remaining three faces. In this situation, Face 9 has the eye gap value that is closest to the middle value of the local context defined by the three faces and thus may seem the most pleasant. Now cover the leftmost face. In this context, Face 13 has the eye gap that is closest to the middle value of the new local context and appears most pleasant. In Experiment 2 we tested this prediction in a between-subjects design.

\section{Method}

Participants were 120 students selected from the same population as before and were randomly assigned to 1 of the 12 conditions resulting from the combination of context (low or high) and arrangement (six counterbalanced arrangements of the faces on the page). The dependent variable was a coding of which face was chosen.

Materials consisted of 2-page booklets. On the first page were printed instructions. On the second page were the three faces, printed in a row. Faces varied in eye gap in the same way as in Experiment 1. The low-range set consisted of Faces 1, 9, and 13, and the high-range set consisted of Faces 9, 13, and 21. Faces were $56 \mathrm{~mm}$ tall and $37 \mathrm{~mm}$ wide.

Participants were tested in groups of 5, with this experiment being the first in a series of decision-making tasks they performed. Instructions informed participants that they were to examine the three faces on the following page and circle the one that appeared most pleasant to look at.

\section{Results}

In both conditions, the extreme contextual face was rarely chosen, constituting only $5.5 \%$ and $3.6 \%$ of choices in the low- and high-range conditions, respectively. In the lowrange condition, Face 9 was chosen by $76.4 \%$ of participants and Face 13 was chosen by only $18.2 \%$ of participants. This preference relationship was reversed in the high-range condition, with $41.1 \%$ choosing Face 9 and $55.4 \%$ choosing Face 13. A log linear analysis of the choice data (excluding the 5 participants who chose the contextual faces) revealed a strong Context $\times$ Target effect, $\chi^{2}(1, N=115)=18.13, p<$ .001 , and no significant interactions with the arrangement variable.

\section{Discussion}

Manipulating the third alternative in the choice set produced a large systematic reversal in choice preferences, consistent with the shifts in ideal-point locations demonstrated in Experiment 1 for single-stimulus judgment tasks. Remarkably, exposure to a single contextual stimulus resulted in an average shift in choice percentages of more than 30 points. These effects point to the lability of ideal points for at least these relatively unfamiliar stimuli.

The choice paradigm used in Experiment 2 resembles that used in the literature on decoy effects in choice. In that literature, adding a third alternative that is rarely chosen can lead to large reversals of preference relationships among the remaining alternatives (Huber, Payne, \& Puto, 1982; Huber \& Puto, 1983; Simonson, 1989; Simonson \& Tversky, 1992; Wedell, 1991; Wedell \& Pettibone, 1996). Although the present manipulation most closely resembled experiments with the compromise decoy, in which an alternative that is extremely good on one dimension but extremely poor on another is added to a choice set, it differs in significant ways. First, unlike all the previous decoy manipulations reported in the literature, the alternatives in Experiment 2 differed on only a single dimension. Second, previous decoy research used monotonic preference domains rather than ideal-point domains. Third, the extreme alternative in Experiment 2 was rarely chosen, but the extreme alternatives making up the compromise decoy are typically chosen much more often (e.g., $10 \%-20 \%$ of the time).

Despite these differences, the results of Experiment 2 may have implications for understanding decoy effects. In the typical decoy paradigm, there are two dimensions that represent a trade-off. For example, Car A may be high on ride quality but low on miles per gallon (mpg), and Car B may be low on ride quality but high on mpg. Although each of these dimensions represents monotonic preference domains, they are combined in the problem in a correlated fashion so that increases in mpg are associated with decreases in ride quality and vice versa. Thus, when examining nondominated choice options, there is a tendency for extremes on a given dimension to be associated with negative values on at least one dimension. Participants may then extract a combined dimension that operates much like an ideal-point preference domain. This extracted dimension may then show similar types of context effects found in ideal-point domains, with the ideal shifting toward the average values in the set of stimuli being considered. The idea of values along an equipreference contour following an ideal-point process is not new; it was proposed by Coombs (1975) in his portfolio theory. We build on this idea by suggesting that the ideal point of combined dimensions may shift with context and contribute to what Simonson and Tversky (1992) referred to as extremeness aversion.

\section{Experiment 3: Effects of Local Context in Choice}

Experiment 2 demonstrated contextual preference reversals in choice that were in line with the idea that ideals shift toward the mean of the contextual stimuli. In Experiment 2 there was only one choice set so that the local context defined by the current choice set was the same as the global context defined by all previously encountered stimuli. Thus, the results of Experiment 2 leave open the question of whether manipulation of the local contextual choice set can lead to shifts in preference even when the global context of all the previous choice sets is equated for participants. In Experiment 3 the global context, as defined by the entire set of faces shown to participants, was held constant. On key trials, the two target faces ( 9 and 13) were presented together on the screen with a third contextual face of a more extreme value. If the context determining ideals is the global context, 
then manipulating the third contextual face in the choice set should have little effect on choice. However, if the ideal point for a given set is strongly influenced by the alternatives within that set, then reversals of preferences such as those found in Experiment 2 should occur.

\section{Method}

Participants and design. Participants were 62 students from the same population used in Experiments 1 and 2. Participants were presented with choice sets composed of three faces that varied only in the gap between the eyes. Target sets were composed of two moderate target faces and one, more extreme, contextual face. Filler sets were composed of three contextual faces. The dependent variable was the face that was selected as most pleasant in each target set. Within-subjects independent variables included the context face (varied at six levels) and the arrangement on the screen (each set was presented in each of six possible arrangements).

Materials and apparatus. Microcomputers were used to present all instructions and stimulus materials and to collect all responses. Faces used in this experiment were identical to those used in the previous experiments. Two moderate eye gap faces ( 9 and 13) were used as target faces. Six extreme faces $(1,5,7,15,17$, and 21) were used as context faces. Each face was presented in every possible unique combination of the eight faces, resulting in 6 target sets involving both target faces and a context face. The remaining 20 sets contained either a single target face or only contextual faces and are thus considered filler sets. Each target set was presented 6 times, once in each possible screen presentation arrangement. Likewise, each filler set was presented in three different presentation arrangements. This resulted in participants receiving 96 choice sets in all. Nose width for all faces was held constant at 30 pixels (Size 6).

Procedure. Participants were tested in groups of 5 in a laboratory with computer terminals spaced about $1 \mathrm{~m}$ apart. After reading the general instructions, participants were shown 96 trinary-choice sets and were told to select the face that they felt was the most pleasant to look at. Faces were presented side by side, equally spaced and aligned on the screen. Each face was the same size. Under each face was printed a large magenta number. To make their choice, participants pressed 1,2, or 3 to select the face on the left, middle, or right, respectively. After they made a choice, a new choice set was presented following a 2 -s delay.

\section{Results}

Figure 3 presents the choice percentages for Faces 9 and 13 as a function of the contextual face included in the choice set. When the contextual face had a narrow eye gap, Face 9 was preferred more than Face 13, but the reverse preference ordering was true when the contextual face had a wide eye gap. This crossover interaction is consistent with the predicted preference reversals; however, the effect is not as large as that found in the between-subjects design of Experiment 2, with an average shift of only 10 points from Contextual Face 1 to Face 21.

We conducted a repeated-measures MANOVA on the choice percentages as a function of target and context. The main effect of context, $F(5,57)=35.6, p<.001$, simply reflected a tendency to choose the contextual face more often for some of the middle-valued faces (7 and 15). The predicted Context $\times$ Target interaction was significant, $F(5$,

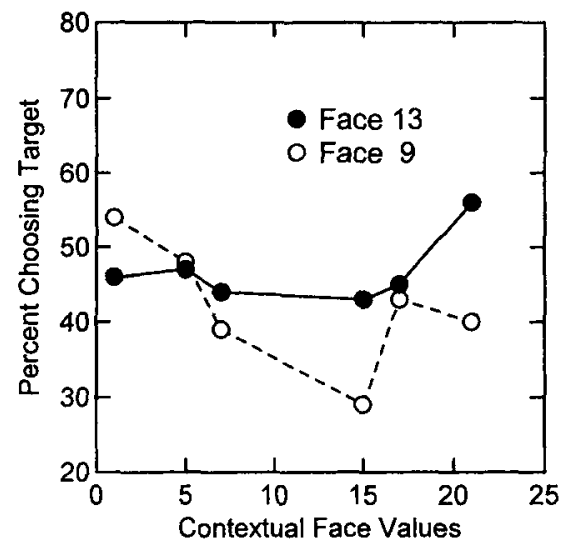

Figure 3. The mean choice percentages from Experiment 3, which provide support for local context effects on choice. Preference for Face 13 over Face 9 is greater when the value of the contextual face is high rather than low. Note that percentages that do not add up to $100 \%$ reflect selection of the contextual face.

$57)=3.3, p<.05$. Polynomial contrasts indicated that the only significant interaction trend was the linear trend, $F(1$, $61)=11.4, p<.001$, indicating an increasing tendency to prefer Face 13 over Face 9 as the eye gap for the contextual face widened.

We calculated an index of predicted preference reversals for each participant by comparing the percentage of choices for the target faces when it was favored by the contextual face versus when it was not favored. The participants averaged $12.2 \%$ reversals.

\section{Discussion}

In Experiment 3, manipulating the third alternative in the choice set produced a small but systematic shift in choice preferences even when the global context of the full set of contextual faces was held constant. This result indicates that the local context can affect choice by presumably affecting the location of the ideal point for that choice set. The reduced magnitude of reversals compared to Experiment 2 is not surprising given that there were a large number of choice sets making up a stable global context. Furthermore, each participant saw the two target faces in each of 36 sets. If they sought to maintain consistent responses, they would be quite reluctant to switch preferences from trial to trial. The combined results of Experiments 2 and 3 are consistent with the typical finding that preference reversals are much larger when the key manipulation is between subjects rather than within subjects (Kuhberger, 1995; Levin, Johnson, \& Davis, 1987).

\section{Experiment 4: Shifting Ideals in a Two-Dimensional Space}

Experiments 1-3 and previous tests of the contextual dependence of ideals (Riskey et al., 1979) examined these effects for unidimensional manipulations of context. In Experiment 4 we simultaneously varied eye gap and nose 
width to determine if the effects observed for unidimensional manipulations would generalize to multidimensional manipulations. The most straightforward prediction is that the effects of the manipulations would be additive so that the ideal in the two-dimensional space would be well predicted by the shifts for each of the separate dimensions. On the other hand, the features might combine interactively, or additional dimensions might emerge, so that a more complex pattern of results is obtained. As in Experiment 1, the pattern of judgments was modeled to gain insight into the nature of the ideal-point process.

\section{Method}

Participants and design. Participants were 114 undergraduates sampled from the same population as Experiments 1-3. The basic design consisted of a $2 \times 2 \times 7 \times 7$ factorial combination of eye gap context, nose width context, eye gap targets, and nose width targets. The target variables were manipulated within subjects, and the contextual variables were manipulated between subjects. The dependent variable was the 9-point rating of pleasantmess of each of the 49 target faces. Order of presentation was randomized for each participant.

Materials and apparatus. Microcomputers were used to present all instructions and stimulus materials and to collect all responses. The materials were essentially the same as those used in Experiment 1, with Values 5, 9, 11, 13, 15, 17, and 21 serving as common target values. We manipulated context for each dimension by including low-range values $(1,2,3,4,6,7,8)$ or high-range values $(18,19,20,22,23,24,25)$. The stimulus design is illustrated in Figure 4. The combination of eye context and nose context resulted in four contextual sets, two in which contextual stimuli were either consistently low (L) range or high $(\mathrm{H})$ range for both feature domains (LL or $\mathrm{HH}$ ) and two in which contexts differed across features (LH and HL). Altogether, each participant rated 196 faces generated by the $14 \times 14$ combination of eye gap and nose width. A core set of 49 faces was common to all distributions. Additionally, the first 14 faces were rated again after all other faces had been presented so that a measure of reliability could be generated for each participant.

Procedure. Participants were tested in groups of 5 . They were instructed that they would be presented with schematic faces that varied in eye gap and nose width. For each face, they were to rate how pleasant the face appeared to them on a 9-point scale. A preview of 14 unrated faces was presented to familiarize the participants with the set. This was followed by the 210 rated trials. Unlike Experiment 1, participants made ratings only of pleasantness and did not rate the width of eye gap and nose width.

\section{Results}

We calculated reliability indices for participants based on the 14 faces that each individual rated twice. The 28 participants who had reliabilities less than .40 were deleted from the following analyses.

Figure 5 presents a contour plot that corresponds to the mean ratings of the 49 target faces. Solid lines represent transitions from whole-number rating values (i.e., 5.0, 6.0, 7.0 , etc.). Dashed lines represent shifts in ratings of .25 of a rating category, and filled circles represent the locations of the target stimuli. The peak regions are all above 7.0 on the rating scales and correspond roughly to the ideal points.
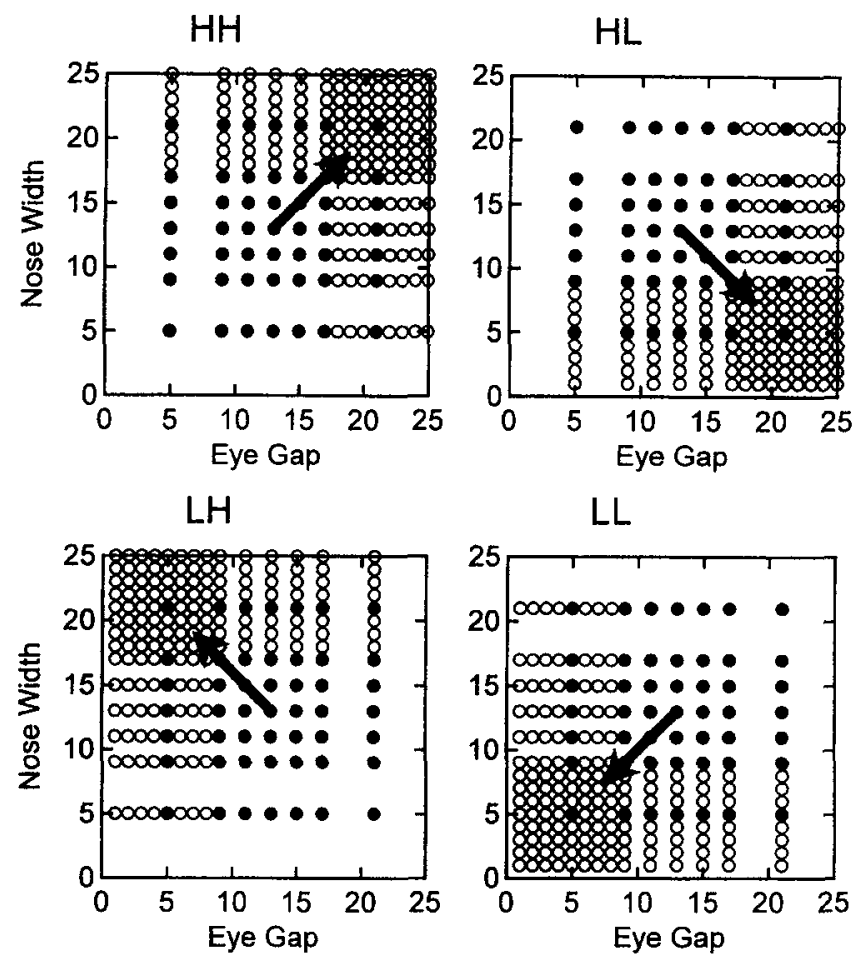

Figure 4. The experimental design of Experiment 4. Filled circles represent locations of core stimuli, and open circles represent locations of contextual stimuli in the two-dimensional space of nose width and eye gap. Core stimuli combine target sizes from both dimensions, whereas contextual stimuli are constructed using one or more of the contextual sizes. The four contextual conditions are high-high (HH), high-low (HL), low-high (LH), and low-low (LL), with the first value representing the eye context and the second representing the nose context. Overall, there are 196 circles for each contextual condition, representing the total number of stimuli rated by each participant. Arrows represent the direction in which the ideal point is predicted to shift for each contextual condition.

Crosshairs represent the values of the ideal point derived from analyses described below. Comparisons of top and bottom panels represent a similar change in the eye gap context. Comparisons of left and right panels represent a similar change in the nose width context. The effects of context shown in Figure 5 are systematic and consistent with the results from Experiment 1. For both eye gap and nose width, the ideal value is higher in the high context than in the low context. Moreover, the effects of the different combinations of contextual ranges appear to be largely independent of one another.

We conducted a $2 \times 2 \times 7 \times 7$ repeated-measures MANOVA on the pleasantness ratings. The main effects of eye targets and nose targets were highly significant $(F \mathrm{~s}>25)$ and simply reflected the fact that there was substantial variation in the rated pleasantness of these faces. More important, the interaction of eye target and eye context was significant, $F(6,77)=9.6, p<.001$, reflecting a downward shift of the ideal eye gap in the low eye gap context. 

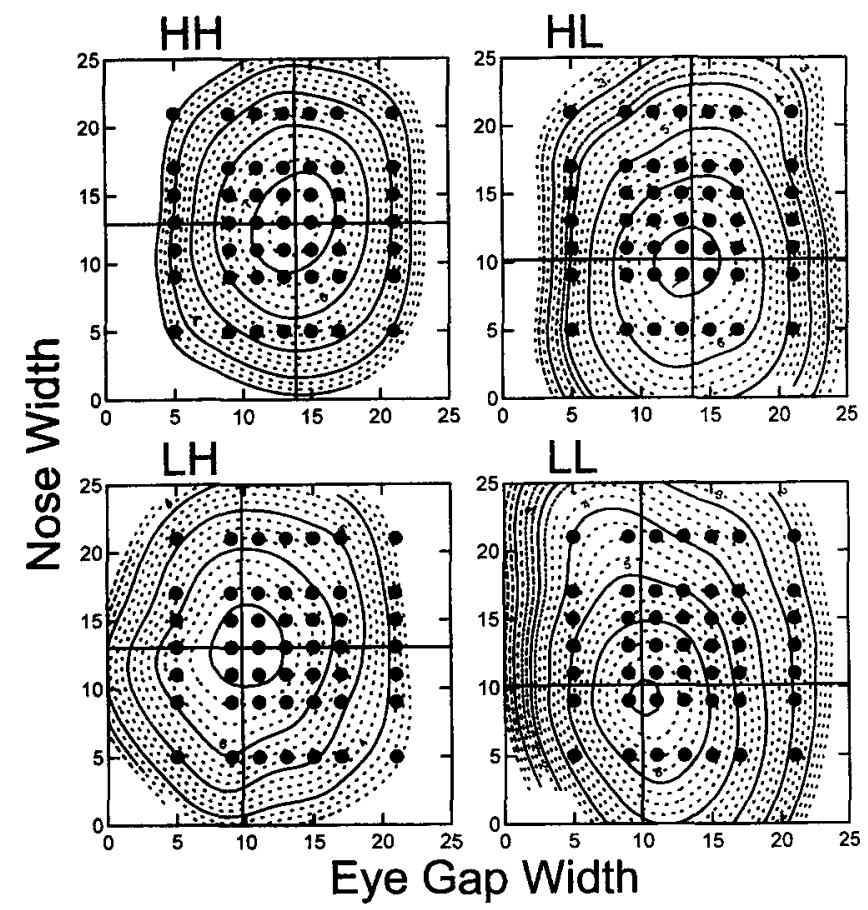

Figure 5. Two-dimensional contour plot of ratings for core stimuli in the high-high (HH), high-low (HL), low-high (LH), and low-low (LL) contexts in Experiment 4. Numbers represent the inferred mean ratings for faces at the solid contour lines. Dashed contours represent a .25 increment in ratings. Crosshairs represent the values of the ideal points as determined by the fit of the data to the ideal-point model.

Similarly, the interaction of nose target and nose context was significant, $F(6,77)=7.2, p<.001$, reflecting a downward shift of the ideal nose width in the low context. The only other significant effect was the interaction of eye target and nose target, $F(36,47)=1.81, p<.05$, which indicated that the contributions of eye gap and nose width to overall ratings of pleasantness were not strictly additive. This is what would be expected given a nonlinear similarity function.

The multidimensional gaussian ideal-point model of Equations 4 and 5 was used to fit the data. The specific equation used was

$$
\mathrm{C}_{\mathrm{ik}}=a+b \exp \left[-c_{\mathrm{k}} \Sigma \mathrm{w}_{\mathrm{m}}\left(\phi_{i m}^{\mathrm{p}}-\mathrm{ideal}_{k m}^{\mathrm{p}}\right)^{2}\right] .
$$

In Equation 8, $w_{m}$ represents the attentional weight given to stimulus dimension $m, c$ once again reflects the narrowness of the function, $a$ is the intercept, and $b$ describes the height of the function. Because there were only two dimensions, a single dimensional weight value was fit, $w_{\text {eyes }}$, with $w_{\text {nose }}=$ $1-w_{\text {eyes }}$. We conducted a nonlinear regression of the stimulus means onto the physical values of eye gap and nose width across the combined contextual sets using Equation 8 and a least squares loss function. The strategy was to initially constrain all parameters to be equal across contexts and then free parameters when they led to a significant increment in $R^{2}$. The best fitting model had 11 free parameters.
Figure 6 graphically presents the fit of the 11-parameter model to the 196 data points ( 4 contextual sets $\times 49$ rated faces), and Table 1 presents the model parameters along with values of $R^{2}$. Note that the response function, as defined by Parameters $a$ and $b$, was constrained to be equal across contexts. Consistent with the independence of the eye gap and nose width contextual manipulations, the fit of the model was not significantly better when ideal points for one dimension were allowed to vary with the contextual manipulation of the other dimension. The only parameter to vary with context other than ideal-point location was the discriminability parameter $\left(c_{\mathrm{k}}\right)$. This variation corresponded to a somewhat narrower ideal-point function for high eye gap contexts than for low eye gap contexts.

\section{Discussion}

The results of Experiment 4 generalize the effects of unidimensional manipulations of context on ideals to multidimensional manipulations. The good fits of the ideal-point model demonstrated the lawfulness of the relationship between preference and context. As the range extended in the multidimensional space to include extreme values along a given dimension, the ideal for that dimension moved toward the extreme stimuli. Thus, when the contextual set consisted of faces with very narrow noses and very narrow eye gaps, the ideal face had a narrower nose and eye gap than when the contextual set consisted of faces with very wide noses and eye gaps.

These data imply multiple preference reversals across contexts. For example, in the HH context, Face 13-13 was preferred to Face 9-9, but the reverse was true in the LL context. In the HL context, Face 13-9 was preferred to Face 9-13, but the reverse was true in the LH context. Such preference reversals across contexts are inconsistent with models that assume monotonic effects of context, monotonic preference relations, or context-invariant ideals in a multidimensional space.

The model fit supported the idea that the effects of manipulating contextual values on one feature were independent of the effects of manipulating contextual value on the other, at least for these two features. Thus, the inferred ideal

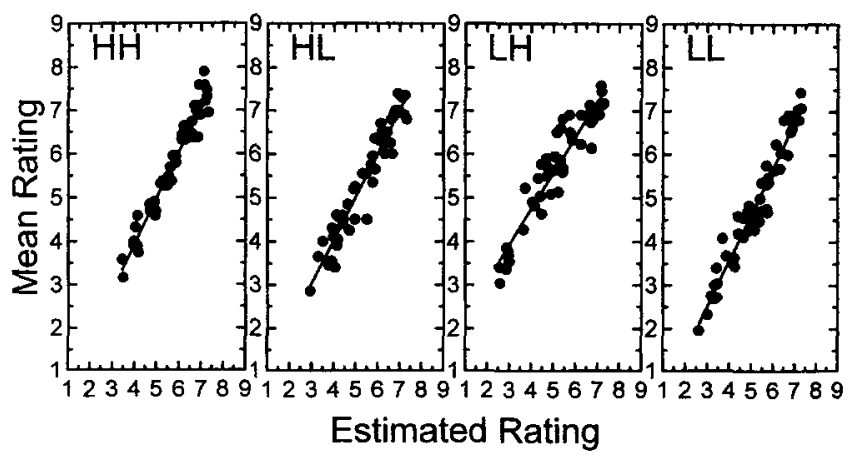

Figure 6. Fit of the gaussian ideal-point model to ratings for core stimuli in the high-high (HH), high-low (HL), low-high (LH), and low-low (LL) contexts in Experiment 4. 
Table 1

Parameters From the Simultaneous Fit of Equation 8 to the Four Contextual Conditions of Experiment 4

\begin{tabular}{ccccccccc}
\hline Context & $a$ & $b$ & $c$ & $\mathrm{w}_{\text {eye }}$ & $p$ & ideal $_{\text {eye }}$ & ideal $_{\text {nose }}$ & $R^{2}$ \\
\hline HH & 1.80 & 5.56 & 0.035 & .661 & .894 & 13.85 & 12.89 & .951 \\
HL & - & - & - & - & - & - & 9.21 & .924 \\
LH & - & - & 0.029 & - & - & 10.24 & 12.89 & .905 \\
LL & - & - & 0.045 & - & - & - & $\underline{9.21}$ & .944 \\
\hline
\end{tabular}

Note. $R^{2}$ is based on a comparison of means to predicted means for each context separately. Dashes indicate that this value was fixed at the same value as the one above it; underlined text indicates that this parameter was fixed at the same value as the matching value in the same column. $\mathrm{HH}=$ high-high, $\mathrm{HL}=$ high-low, $\mathrm{LH}=$ low-high, and LL = low-low, where the first letter designates eye gap context and the second letter designates nose width context.

nose width depended only on the contextual distribution of nose widths and not on the distribution of eye widths. This conclusion is supported by the lack of interactions between targets on one feature and the contextual manipulation of the other feature. It seems unlikely that such independence is generally the case. For example, manipulation of the width of the head might affect the perception of the width of the eye gap or nose width. Future research might be directed toward how ideals are determined for such potentially interactive or configural dimensions.

\section{General Discussion}

Experiments 1-4 demonstrated the pervasive effects of context on the locations of ideals for schematic faces. In Experiment 1, contextual shifts in descriptive ratings of facial features were accompanied by corresponding shifts in the preferred value of the features. Both effects were quite lawful. Context effects on the descriptive dimensions conformed to predictions from Parducci's (1995) rangefrequency theory. Pleasantness ratings were well described by a gaussian ideal-point model in which the ideal corresponded roughly to the scale value rated neutral or moderate on the contextually altered descriptive scale. Experiment 2 generalized these results to a choice situation in which the context was altered by inclusion of a third, rarely chosen alternative in the choice set. The contextually based preference reversals of Experiment 2 were replicated in Experiment 3 in a situation where the global context (defined as the full set of faces) was held constant but the local context (defined as the contextual face included in a given choice set) was manipulated within subjects. Finally, the results of Experiment 4 showed systematic effects of manipulating the multidimensional context on ratings of pleasantness that were in line with the results of the unidimensional manipulations of Experiment 1.

These results build on previous research showing contextual effects on perceptions of the pleasantness of drinks varying in sweetness (McBride, 1985; Riskey et al., 1979), musical pieces varying in tempo (Holbrook \& Anand, 1990), and rooms varying in size dimensions (Baird et al., 1978). We extended this research by demonstrating similar effects of context on ideals for (a) evaluations of faces, a socially relevant domain; (b) both judgment and choice tasks; and (c) both unidimensional and multidimensional manipulations of context. Furthermore, we demonstrated how these effects were well described by a gaussian ideal-point model.

\section{Contextual Processes Determining Ideals}

The effects demonstrated here may seem similar to familiarity effects on attractiveness (Moreland \& Zajonc, 1982 ) in which mere exposure to a stimulus leads to greater preference for that stimulus. However, in our experiments the targets were presented equally often across contexts so that frequency of exposure did not differ. Thus, it is difficult to explain these effects in terms of changes in the familiarity of the target faces. Instead, there are two basic ways to conceptualize these results. The first is that judgment processes operating on the descriptive dimensions cause changes in perceived pleasantness. According to this model, contextual stimuli are recruited at the time of judgment and are used to assess the features along descriptive dimensions (such as width). Range-frequency processes guide these assessments so that the same nose might seem wide in one context but narrow in another. Pleasantness or preference is then mapped onto the contextually altered descriptive ratings. Thus, in the low context a lower value is rated neutral, and the ideal, which is equated with a neutral value, is shifted downward accordingly. In this framework the assimilation effect on the ideal is mediated by a contrast effect on the dimensional judgments. The correspondence between context effects on descriptive and pleasantness judgments in Experiment 1 provides some support for this interpretation, which we refer to as the judgment-mediated model.

An alternative interpretation is that ideals simply reflect the nature of the underlying category structure. Exposure to the set of faces provides the basis for forming a categorical representation. Many aspects of categorical representations can be well described by assuming that a prototype is extracted to represent the concept (Homa, Burruel, \& Field, 1987; Posner \& Keele, 1968). Prototypes tend to fall near the average of stimulus features. According to this prototypemediated model, the ideal is equated with the prototypical values of the category. When people judge pleasantness or preference, they extract the prototype from memory and compare the stimulus with it. Manipulation of context shifts 
the ideal by changing the category average and hence the prototype.

Our experiments do not distinguish between judgmentmediated and prototype-mediated models of these effects. Evidence for the prototype-mediated model would consist of showing clear dissociations between descriptive judgments and pleasantness judgments. Evidence for the judgmentmediated model would hinge on demonstrating more directly how contextual effects on dimensional judgments are reflected in corresponding effects on pleasantness ratings. Future research may distinguish which model best explains the strong contextual sensitivity of ideals implied by our results.

\section{The Nature of Attractiveness}

As noted earlier, our results for the pleasantness of schematic faces may be related to the general finding that the average of a group of faces is preferred to the individual faces (Langlois \& Roggman, 1990). The finding that an averaged face is more attractive than individual faces has been replicated across multiple cultures, including Chinese, Nigerian, Indian, and New Zealand students (Pollard, 1995). Jones and Hill (1993) also demonstrated this effect with populations from Brazil, the United States, Russia, and Venezuelan Hiwi Indians. This cross-cultural evidence provides further support that attractiveness is guided by a process that places the ideal toward the average of the relevant features or dimensions.

Langlois and Roggman's (1990) interpretation of this result follows the prototype model of ideals, in which the categorical representation of faces may include a prototype that represents the average of instances, and it is this prototype that constitutes the ideal. Our research provides a different approach to the understanding of attractiveness that emphasizes how the ideal may depend on the recent set of faces experienced. Within the prototype approach, these contextual effects are conceptualized as the ideal shifting toward the average of the most recently presented context. Contextual effects on ideals suggest that to the extent cultures differ in their exposure to faces with different feature values, the ideal face should shift across cultures.

Although studies of judgments of physical attractiveness have found strong agreement across cultures (Bernstein et al., 1982; Cunningham et al., 1995; Thakerar \& Iwawaki, 1979), these correlational approaches may be relatively insensitive to the manipulation of context. A stronger test would be to sample representatively faces from different cultures, merging the samples into a prototypical face. Such faces would presumably all be attractive (as people across cultures share many of the same features), but they would also represent the distinctive differences across cultures. Individuals from different cultures could rate the attractiveness of these different cultural prototypes. We would predict that people across cultures would agree that all these faces were attractive but that people should show a tendency to find averaged faces from their own culture most attractive, as these would be representative of their cultural context.

More generally, it has long been noted that the media may skew our perceptions of what is attractive. Recently, researchers have focused on how a "thin ideal" is being promoted in the media by the use of models who are typically much thinner than the general population (Stice, 1994; Stice \& Shaw, 1994). The contextual shifts in ideals presented in our research provide a simple psychological basis for how the distribution of body types to which people are repeatedly exposed may shift the ideal. Just as experiencing many faces with very thin noses shifted preference toward a thinner nose (Experiments 1 and 4), constant media bombardment of ultrathin models may shift the ideal body type to a thinner ideal. The consequences of adhering to an impossibly thin body type ideal can be devastating, as reflected in the greater tendency of women to experience depression and the eating disorders of anorexia nervosa and bulimia as well as dissatisfaction with their body image (McCarthy, 1990; Stice, Schupak, Shaw, \& Stein, 1994). Although clearly other processes may be operating in the construction of the thin ideal, the concept of the contextual basis of ideals implies that a shift toward presenting less thin body types in the media should likewise shift the ideal to a more moderate and attainable body type.

\section{Context and Attitude}

The single-peaked pleasantness functions shown in the bottom panels of Figure 2 are similar in form to what one finds in plotting an individual's endorsement of attitudes that vary along a continuum (Sherif \& Hovland, 1961). The ideal point in this instance would be considered the individual's attitude. The contextual shift in ideal-point locations can then be considered an index of overall attitude change. In the present set of experiments, attitudes about the schematic faces shifted toward the values of the contextual faces presented, demonstrating an assimilation effect. This type of change is not explained by several standard models of attitude change (see Hunter, Danes, \& Cohen, 1984, for a formalization of seven basic attitude models). For example, models based on balance, congruity, dissonance, or consistency all hinge on the concept of a similarity comparison with or favorability assessment of the source of the message. Source information appears irrelevant in the present experimental paradigm, in which faces were simply presented repeatedly for judgment. Likewise, reinforcement theories appear irrelevant to the current experimental paradigm. Social judgment theory (Sherif \& Hovland, 1961) predicts an assimilation effect when attitudes close to one's own are presented but either no change or a boomerang (contrast) effect when distant attitudes are presented. Because the contextual faces were mostly very distant from the preferred faces, the present results do not appear to be consistent with social judgment theory predictions.

Indeed, the effects demonstrated here seem to lie outside standard theories of attitude change. The results seem most similar to the attitude-change experiments that reflect mere exposure effects (Moreland \& Zajonc, 1982). In those experiments there is little in the way of reasoning or balancing of attitudes toward source and message. Rather, repeated exposure appears to build familiarity (subjectively 
detected as greater processing fluency), and familiarity is positively valued (Grush, 1980). Similarly, in Experiments 1 and 4, the mere exposure to faces at one extreme or the other influenced the attitudes toward the target faces, presumably without consideration of issues regarding source or message discrepancy.

The contextual basis of ideals demonstrated in Experiments $1-4$ suggests a simple mechanism that may underlie some of the basic relationships found between an individual's attitude and attitudes of others. For example, propinquity effects (Newcomb, 1961), in which people tend to have attitudes similar to those who live closest to them, would be expected if one's attitude shifted toward the average of the attitudes to which one was regularly exposed. Similarly, peer group effects (Miller \& Prentice, 1994) may be due in part to this simple mechanism, as attitudes of peers may constitute the largest contributor to the distribution of attitudes to which one is exposed. Indeed, the simple nature of the demonstrated context effects may argue for their widespread applicability.

On the other hand, one may question to what extent these effects will occur in attitudes that have propositional representations. The clearest demonstrations of contextual shifts in ideals have been made with perceptual stimuli: faces in the present experiments, sweetness of drinks (McBride, 1985; Riskey et al., 1979), musical tempo (Holbrook \& Anand, 1990), and architectural dimensions (Baird et al., 1978). In large part, these stimuli are perceptually or directly experienced, as compared to attitudes about positions that are supported by more complex configurations of interrelated propositions and reasons (e.g., attitudes toward abortion, welfare, education, etc). It is possible that the more an attitude is based on a propositional network of reasons, the less likely it will be shifted by exposure to extreme contextual stimuli. In such cases, mechanisms associated with traditional theories of attitude change, such as cognitive consistency, cognitive dissonance, and congruity among beliefs, will become dominant.

The correspondence between descriptive and evaluative judgments demonstrated in Experiment 1 suggests that one may be able to shift attitudes in domains in which one can achieve shifts in the perceptions of different positions. In domains in which one finds little shift in perceptions of attitude objects as a function of context, one should likewise find little shift in preference or attitude. However, there is an accumulating body of research that suggests our preferences may be constructed at the time of choice or judgment and therefore are susceptible to contextual effects. Preference reversals for consumer products can easily be induced by changing the recent set of contextual experiences (Mellers \& Cooke, 1994; Simonson, 1989; Simonson \& Tversky, 1992; Wedell, 1991, 1998; Wedell \& Pettibone, 1996), and thus a promising domain for future research on the contextual dependence of ideals is in the area of consumer attitudes and preferences.

A particularly fruitful domain for future study might be attitudes toward politicians. Such attitudes appear to exhibit a great deal of lability, with large shifts in approval ratings possible over short periods of time. The conventional wisdom of "spin doctors" seems consistent with some of the effects and mechanisms we have described. For example, if Candidate $\mathbf{A}$ is moderately liberal and Candidate $\mathbf{B}$ is moderately conservative, what will be the effect of a viable third candidate, Candidate $\mathrm{L}$, who is much more liberal than A? Insofar as candidate representations are complex enough that voters do not simply retrieve evaluations of them, the inclusion of $\mathrm{L}$ should make $\mathrm{A}$ appear more moderate and $\mathrm{B}$ appear more extreme. If the ideal candidate is moderate, then $A$ should benefit more from comparisons to $L$ than $B$ will. This relationship appears to be endorsed by conventional wisdom, but we know of no formal tests of this prediction.

\section{References}

Anderson, N. H. (1981). Foundations of information integration theory. New York: Academic Press.

Baird, J. C., Cassidy, B., \& Kurr, J. (1978). Room preference as a function of architectural features and user activities. Journal of Applied Psychology, 63, 719-727.

Bernstein, I. H., Lin, T. D., \& McClellan, P. (1982). Cross- vs. within-racial judgments of attractiveness. Perception \& Psychophysics, 32, 495-503.

Berscheid, E., \& Walster, E. (1974). Physical attractiveness. In L. Berkowitz (Ed.), Advances in experimental social psychology (Vol. 7, pp. 157-215). New York: Academic Press.

Birnbaum, M. H. (1974). Using contextual effects to derive psychophysical scales. Perception \& Psychophysics, 15, 89-96.

Boring, E. G. (1950). A history of experimental psychology (2nd ed.). Englewood Cliffs, NJ: Prentice Hall.

Coombs, C. H. (1964). A theory of data. New York: Wiley.

Coombs, C. H. (1975). A note on the relation between the vector model and the unfolding model for preferences. Psychometrika, $40,115-116$.

Coombs, C. H., \& Avrunin, G. S. (1977). Single-peaked functions and the theory of preference. Psychological Review, 84, 216230.

Cunningham, M. R., Roberts, A. R., Barbee, A. P., Druen, P. B., \& Wu, C.-H. (1995). "Their ideas of beauty are, on the whole, the same as ours": Consistency and variability in the cross-cultural perception of female physical attractiveness. Journal of Personality and Social Psychology, 68, 261-279.

Eagly, A., \& Chaiken, S. (1993). The psychology of attitudes. Orlando, FL: Harcourt Brace Jovanovich.

Grush, J. E. (1980). Impact of candidate expenditures, regionality, and prior outcomes on the 1976 Democratic presidential primaries. Journal of Personality and Social Psychology, 38, 337-347.

Holbrook, M. B., \& Anand, P. (1990). Effects of tempo and situational arousal on the listener's perceptual and affective responses to music. Psychology of Music, 18, 150-162.

Homa, D., Burruel, L., \& Field, D. (1987). The changing composition of abstracted categories under manipulations of decisional change, choice difficulty, and category size. Journal of Experimental Psychology: Learning, Memory, and Cognition, 13, 401-412.

Huber, J., Payne, J. W., \& Puto, C. (1982). Adding asymmetrically dominated alternatives: Violations of regularity and the similarity hypothesis. Journal of Consumer Research, 9, 90-98.

Huber, J., \& Puto, C. (1983). Market boundaries and product choice: Illustrating attraction and substitution effects. Journal of Consumer Research, 10, 31-44.

Hunter, J. E., Danes, J. E., \& Cohen, S. H. (1984). Mathematical 
models of attitude change. Volume 1: Change in single attitudes and cognitive structure. Orlando, FL: Academic Press.

Jones, D., \& Hill, K. (1993). Criteria of facial attractiveness in five populations. Human Nature, 4, 271-296.

Kuhberger, A. (1995). The framing of decisions: A new look at old problems. Organizational Behavior and Human Decision Processes, 62, 230-250.

Langlois, J. H., \& Roggman, L. A. (1990). Attractive faces are only average. Psychological Science, 1, 115-121.

Langlois, J. H., Roggman, L. A., \& Musselman, L. (1994). What is average and what is not about attractive faces. Psychological Science, 5, 214-220.

Levin, I. P., Johnson, R. D., \& Davis, M. L. (1987). How information frame influences risky decisions: Between-subjects and within-subjects comparisons. Journal of Economic Psychology, 8, 43-54.

Manis, M. C. (1967). Context effects in communication. Journal of Personality and Social Psychology, 5, 326-334.

McBride, R. L. (1985). Stimulus range influences intensity and hedonic ratings of flavour. Appetite, 6, 125-131.

McCarthy, M. (1990). The thin ideal, depression and eating disorders in women. Behaviour Research and Therapy, 28, 205-215.

Mellers, B. A. (1983). Equity judgment: A revision of Aristotelian views. Journal of Experimental Psychology: General, 111, 242-270.

Mellers, B. A. (1986). "Fair" allocations of salaries and taxes. Journal of Experimental Psychology: Human Perception and Performance, 12, 80-91.

Mellers, B. A., \& Birnbaum, M. H. (1982). Loci of contextual effects in judgment. Journal of Experimental Psychology: Human Perception and Performance, 8, 582-601.

Mellers, B. A., \& Birnbaum, M. H. (1983). Contextual effects in social judgment. Journal of Experimental Social Psychology, 19, 157-171.

Mellers, B. A., \& Cooke, A. D. J. (1994). Tradeoffs depend on attribute range. Journal of Experimental Psychology: Human Perception and Performance, 20, 1055-1067.

Miller, D. T., \& Prentice, D. A. (1994). Collective errors and errors about the collective. Personality and Social Psychology Bulletin, 20, 541-550.

Moreland, R. L., \& Zajonc, R. B. (1982). Exposure effects in person perception: Familiarity, similarity, and attraction. Journal of Experimental Social Psychology, 18, 395-415.

Moskowitz, H. R., Kluter, R. A., Westerling, J., \& Jacobs, H. L. (1974, May). Sugar sweetness and pleasantness: Evidence for different psychological laws. Science, 184, 583-585.

Newcomb, T. M. (1961). The acquaintance process. New York: Holt, Rinehart \& Winston.

Nosofsky, R. M. (1986). Attention, similarity, and the identificationcategorization relationship. Joumal of Experimental Psychology: General, 115, 39-57.

Nosofsky, R. M. (1992). Exemplar-based approach to relating categorization, identification, and recognition. In F. G. Ashby (Ed.), Multidimensional models of perception and cognition (pp. 363-393). Hillsdale, NJ: Erlbaum.

Parducci, A. (1965). Category judgments: A range-frequency model. Psychological Review, 72, 407-418.

Parducci, A. (1995). Happiness, pleasure and judgment: The contextual theory and its applications. Mahwah, NJ: Erlbaum.

Parducci, A., \& Wedell, D. H. (1986). The category effect with rating scales: Number of categories, number of stimuli, and method of presentation. Journal of Experimental Psychology: Human Perception and Performance, 12, 496-516.
Pollard, J. S. (1995). Attractiveness of composite faces: A comparative study. International Journal of Comparative Psychology, $8(2), 77-83$.

Posner, M. I., \& Keele, S. W. (1968). On the genesis of abstract ideas. Journal of Experimental Psychology, 77, 353-363.

Posner, M. I., \& Keele, S. W. (1970). Retention of abstract ideas. Joumal of Experimental Psychology, 83, 304-308.

Riskey, D. R., Parducci, A., \& Beauchamp, G. K. (1979). Effects of context in judgments of sweetness and pleasantness. Perception \& Psychophysics, 26, 171-176.

Rosch, E., \& Mervis, C. B. (1975). Family resemblances: Studies in the internal structure of categories. Cognitive Psychology, 7, 573-605.

Sherif, M., \& Hovland, C. I. (1961). Social judgment: Assimilation and contrast effects in communication and attitude change. New Haven, CT: Yale University Press.

Simonson, I. (1989). Choice based on reasons: The case of attraction and compromise effects. Journal of Consumer Research, 16, 158-174.

Simonson, I., \& Tversky, A. (1992). Choice in context: Tradeoff contrast and extremeness aversion. Journal of Marketing Research, 29, 281-295.

Smith, R. H., Diener, E., \& Wedell, D. H. (1989). Intrapersonal and social comparison determinants of happiness: A range-frequency analysis. Journal of Personality and Social Psychology, 56, 317-325.

Stice, E. (1994). Review of the evidence for a sociocultural model of bulimia nervosa and an exploration of the mechanisms of action. Clinical Psychology Review, 14, 633-661.

Stice, E., Schupak, N. E., Shaw, H. E., \& Stein, R. I. (1994). Relation of media exposure to eating disorder symptomatology: An examination of mediating mechanisms. Journal of Abnormal Psychology, 103, 836-840.

Stice, E., \& Shaw, H. E. (1994). Adverse effects of the media portrayed thin-ideal on women and linkages to bulimic symptomatology. Joumal of Social and Clinical Psychology, 13, 288308.

Thakerar, J. N., \& Iwawaki, S. (1979). Cross-cultural comparisons in interpersonal attraction of females toward males. Journal of Social Psychology, 108, 121-122.

Thurstone, L. L., \& Chave, E. J. (1929). The measurement of attitude. Chicago: University of Chicago Press.

Wedell, D. H. (1991). Distinguishing among models of contextually induced preference reversals. Journal of Experimental Psychology: Learning, Memory, and Cognition, 17, 767-778.

Wedell, D. H. (1995). Contrast effects in paired comparisons: Evidence for both stimulus-based and response-based processes. Joumal of Experimental Psychology: Human Perception and Performance, 21, 1158-1173.

Wedell, D. H. (1996). A constructive-associative model of the contextual dependence of unidimensional similarity. Journal of Experimental Psychology: Human Perception and Performance, 22, 634-661.

Wedell, D. H. (1998). Testing models of trade-off contrast in pairwise choice. Journal of Experimental Psychology: Human Perception and Performance, 24, 49-65.

Wedell, D. H., \& Parducci, A. (1988). The category effect in social judgment: Experimental ratings of happiness. Journal of Personality and Social Psychology, 58, 319-329.

Wedell, D. H., Parducci, A., \& Geiselman, R. E. (1987). A formal analysis of ratings of physical attractiveness: Successive contrast and simultaneous assimilation. Journal of Experimental Social Psychology, 23, 230-249. 
Wedell, D. H., Parducci, A., \& Lane, M. (1990). Reducing the dependence of clinical judgment on the immediate context: Effects of number of categories and type of anchors. Journal of Personality and Social Psychology, 58, 319-329.

Wedell, D. H., Parducci, A., \& Roman, D. (1989). Student perceptions of fair grading: A range-frequency analysis. American Journal of Psychology, 102, 233-248.
Wedell, D. H., \& Pettibone, J. C. (1996). Using judgments to understand decoy effects in choice. Organizational Behavior and Human Decision Processes, 67, 326-344.

Received July 12, 1998 Revision received October 20, 1998 Accepted October 20, 1998

\section{Call for Nominations}

The Publications and Communications Board has opened nominations for the editorships of Behavioral Neuroscience, JEP: Applied, JEP: General, Psychological Methods, and Neuropsychology for the years 2002-2007. Michela Gallagher, PhD; Raymond S. Nickerson, PhD; Nora S. Newcombe, PhD; Mark I. Appelbaum, PhD; and Laird S. Cermak, PhD, respectively, are the incumbent editors.

Candidates should be members of APA and should be available to start receiving manuscripts in early 2001 to prepare for issues published in 2002. Please note that the P\&C Board encourages participation by members of underrepresented groups in the publication process and would particularly welcome such nominees. Self-nominations are also encouraged.

To nominate candidates, prepare a statement of one page or less in support of each candidate. The search chairs are as follows:

- Joe L. Martinez, Jr., PhD, for Behavioral Neuroscience

- Lauren B. Resnick, PhD, and Margaret B. Spencer, PhD, for JEP: Applied

- Sara B. Kiesler, PhD, for JEP: General

- Lyle E. Bourne, Jr., PhD, for Psychological Methods

- Lucia A. Gilbert, PhD, for Neuropsychology

Address all nominations to the appropriate search committee at the following address:

[Name of journal] Search Committee clo Karen Sellman, P\&C Board Search Liaison Room 2004

American Psychological Association 750 First Street, NE

Washington, DC 20002-4242

The first review of nominations will begin December 6, 1999. 